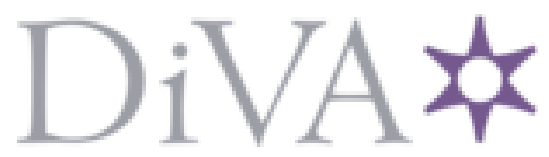

http://www.diva-portal.org

\title{
Postprint
}

This is the accepted version of a paper published in Applied Mechanics and Materials. This paper has been peer-reviewed but does not include the final publisher proof-corrections or journal pagination.

Citation for the original published paper (version of record):

Bocko, J., Dorn, M., Nohajová, V. (2015)

Application of Evolutionary Algorithm in Elasticity.

Applied Mechanics and Materials, 816: 363-368

http://dx.doi.org/10.4028/www.scientific.net/AMM.816.363

Access to the published version may require subscription.

N.B. When citing this work, cite the original published paper.

Permanent link to this version:

http://urn.kb.se/resolve?urn=urn:nbn:se:Inu:diva-46760 


\section{Application of Evolutionary Algorithm in Elasticity}

Jozef BOCKO ${ }^{a}$, Michael DORN ${ }^{b}$, Viera NOHAJOVÁc

${ }^{a}$ Department of Applied Mechanics and Mechanical Engineering, Faculty of Mechanical

Engineering, Technical University of Košice, Letná 9, Košice, 04200 Košice, Slovakia. jozef.bocko@tuke.sk

${ }^{b}$ Department of Building Technology, Linnaeus University, 35195 Växjö, Sweden. michael.dorn@Inu.se

${ }^{c}$ Department of Applied Mechanics and Mechanical Engineering, Faculty of Mechanical

Engineering, Technical University of Košice, Letná 9, Košice, 04200 Košice, Slovakia. viera.nohajova@tuke.sk

Applied Mechanics and Materials, 816:363-368, 2015.

http://dx.doi.org/10.4028/www.scientific.net/AMM.816.363

Available under the conditions of Blue open access as the post-print version of the accepted article. See also http://www.sherpa.ac.uk/romeo/search.php?issn=1660-9336 


\title{
Application of Evolutionary Algorithm in Elasticity
}

\author{
Bocko Jozef ${ }^{1, a}$, Dorn Michael ${ }^{2, b}$, Nohajová Viera ${ }^{1, c^{*}}$ \\ ${ }^{1}$ Department of Applied Mechanics and Mechanical Engineering, Faculty of Mechanical \\ Engineering, Technical University of Košice, Letná 9, Košice, 04200 Košice, Slovakia \\ 2Department of Building Technology, Linnaeus University, 35195 Växjö, Sweden \\ ajozef.bocko@tuke.sk, bmichael.dorn@Inu.se, viera.nohajova@tuke.sk
}

Keywords: Evolutionary algorithm, Bodner-Partom model, parameters, stress-strain curve.

\begin{abstract}
This article introduces evolutionary algorithms and their utilization in mechanical engineering. First part of this work describes evolutionary algorithms and their characteristics. The main body of evolutionary algorithms, the selection methods for parents and the types of reproduction are explained in the next part of this article. Termination conditions are also discussed. Finally, the application of evolutionary algorithms to a problem in mechanical engineering is described. Thereby, the material parameters for a Bodner-Partom model describing viscoelastoplastic material behavior are determined by fitting data from experiments on Aluminum test samples under tension load.
\end{abstract}

\section{Introduction}

Evolutionary algorithms have become a matter of interest in recent years. Routing and scheduling tasks, movement planning of robots and various computer programs use the advantages of evolutionary algorithms. The final solution to a problem depends on an initial set of random candidate solutions, improving themselves to minimize the error and finally finding the optimum. Evolutionary algorithms are becoming more popular for solving problems with very difficult mathematical models and boundary conditions. They are performing well even when common optimization methods are not practicable or too complicated.

\section{Evolutionary algorithms}

By the term evolutionary algorithms usually three methods of problem solving are summarized which are developed independently of each other:

- Evolutionary strategy

- Evolutionary programming

- Genetic algorithms.

Evolutionary strategy is a method which is based on monitoring the influence of random mutations on the designed system. Every individual creates its own model of function of error. The individual uses this model for self-adaptation. The chromosome includes the parameters of the object and the strategy parameters. Both parts of the chromosome are exposed to the mutation.

Evolutionary programming uses random mutations during evolution. It is the oldest type of evolutionary algorithms and was developed in the half of 20-th century. Mutation is used for very small to very drastic changes to reach the optimum. The selection is performed by tournaments.

Most popular are genetic algorithms, a case of machine learning that is based on the theory of artificial life. Roulette methods are used for the selection of parents for one-point or multi-point crossovers. Individuals have the form of a vector of parameters.

At the beginning of the evolution process, the individuals have rules that solve the general problem in the first generation. During the evolution they gain the experience and improve the rules to solve specific problems. System searches the most appropriate set of rules for the situation. 
A special type of genetic algorithms is genetic programming. The chromosome has a tree structure, not a sequence used in common genetic algorithms. The most used genetic operator in genetic programming is crossover. The selection of individuals is performed by the roulette method, by tournaments or by random selection $[7,9]$.

\section{The body of evolutionary algorithms}

Evolutionary algorithms are deployed to solve different types of equations. At the beginning, the first population of individuals is created, also called "the population of zeroth generation". Genetic operators are then used to create new individuals and they depend on the type of properties in the parent's chromosomes. Properties of the most suitable solutions of the problem are transferred to the next generation $[1,2,5,8]$. By this, the individuals are repeatedly changed and improved during the run. The general body of evolutionary algorithms is shown in Fig. 1:

- Initialization

- Selection

- Genetic operators

- Evaluation

- Termination condition.

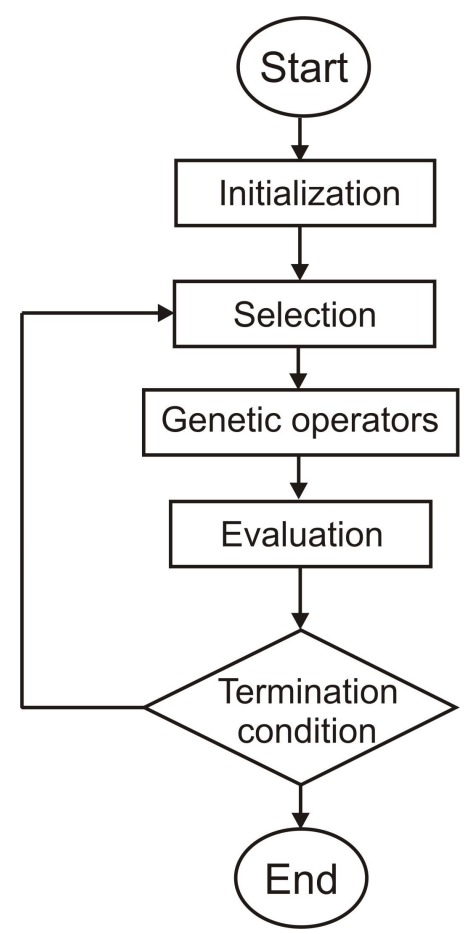

Fig. 1 General Structure of Evolutionary Algorithm

The initialization of the population of the zeroth generation is the first part in the cycle of an evolutionary algorithm. The first population is created using random values. When the individuals in the first generation are not sufficiently good to solve the problem, the selection process follows. The best individuals for reproduction are chosen and become parents for new individuals. Basically, there are two types of selection: soft and hard selection. In soft selection, every individual will have the same chances to become a parent including above and below average individuals. In hard selection, only the most suitable individuals will become parents.

The most used methods during selection are amongst others, the roulette method, random selection and tournaments. Reproduction is done by so-called genetic operators, either crossover or mutation. Crossover is a sexual operator whereby the genetic material of both parents is mixed and inherited by the child (Figs. 2-5). Mutation, in contrast, is an asexual operator. The child has just one parent, but one or more elements are changed during reproduction (Fig. 6). 
Usually, crossover influences the evolutionary processes more than mutation therefore crossover is more widely used to create a new individuals. Still, since the set genetic material of the total population is limited, mutation is essential to introduce new genetic material. Different methods for crossover are available [5], including:

- One-point crossover (Fig. 2) - A single point is chosen where the parent individuals are divided in two parts. By this, two parents can create two different children by cross-wise combination of the parts of the parents.

- Multi-point crossover (Fig. 3) - The parents are divided by more than one point and the children are created by replacing respective parts of the parents' chromosomes.

- Uniform crossover (Fig. 4) - This type of crossover is also known as scattered crossover. A random binary string (the mask) is created with the same length as the chromosomes of the parents. The crossover uses information of the mask to select material from either of the parents.

- Arithmetic crossover (Fig. 5) - The chromosomes of both parent are modified by weighing functions and then summed up to create the new individual.

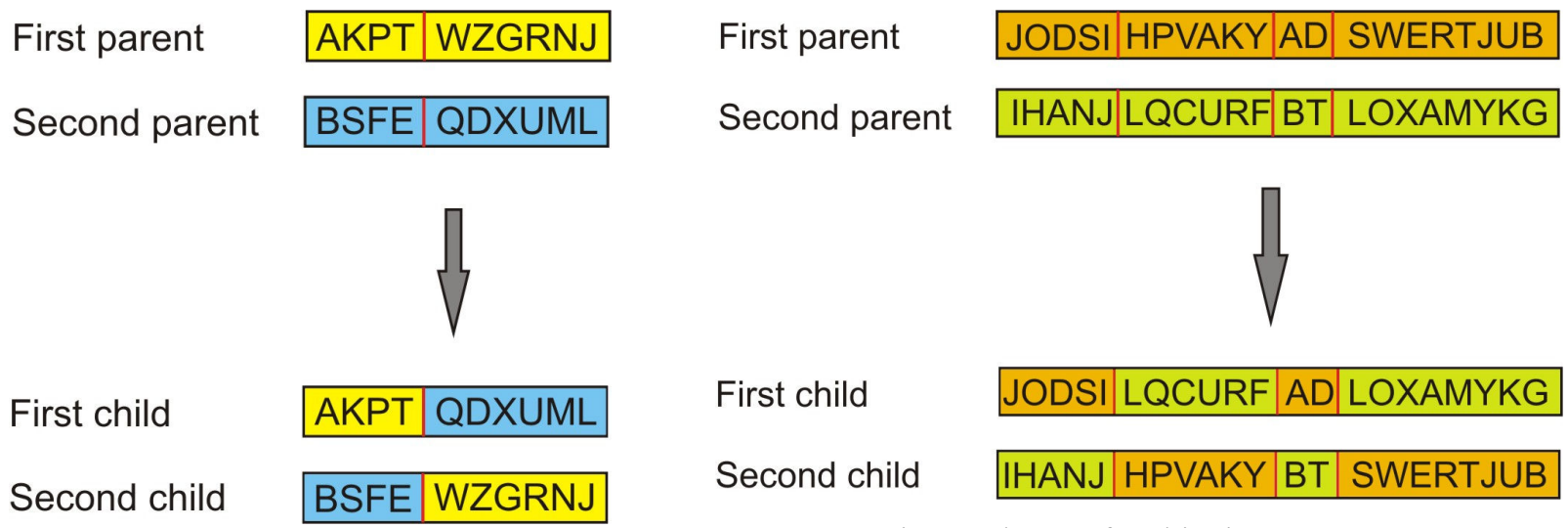

Fig. 2 Scheme of one-point crossover

Fig. 3 Scheme of multipoint crossover

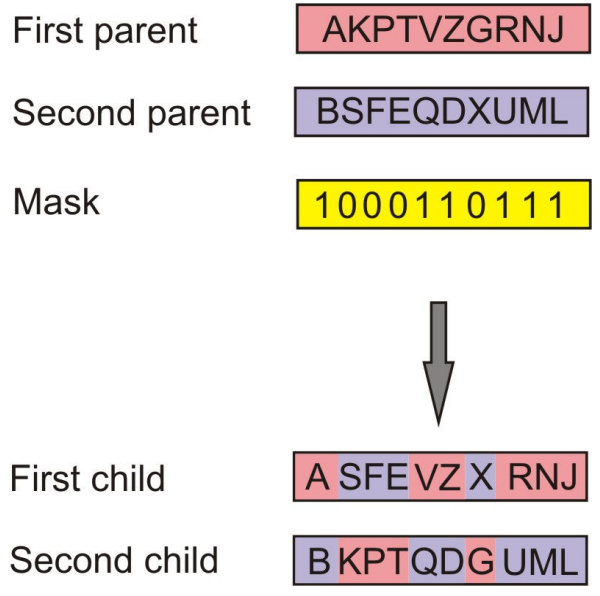

Fig. 4 Scheme of uniform crossover

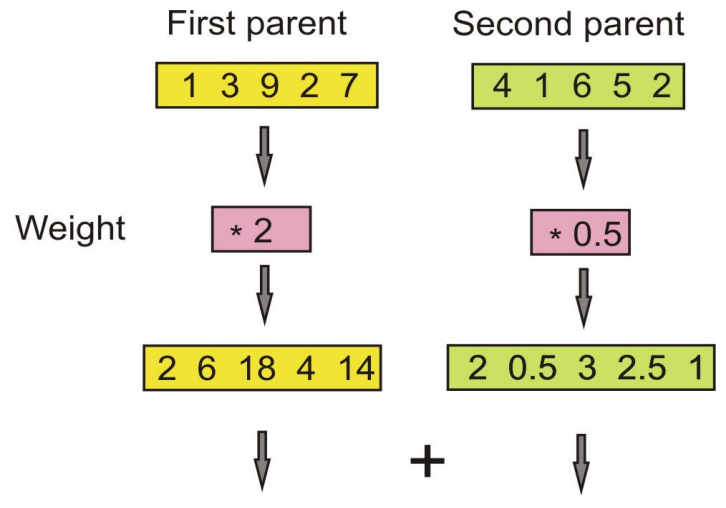

First child $\quad 4 \quad 6.521 \quad 6.5 \quad 15$

Fig. 5 Scheme of arithmetic crossover 


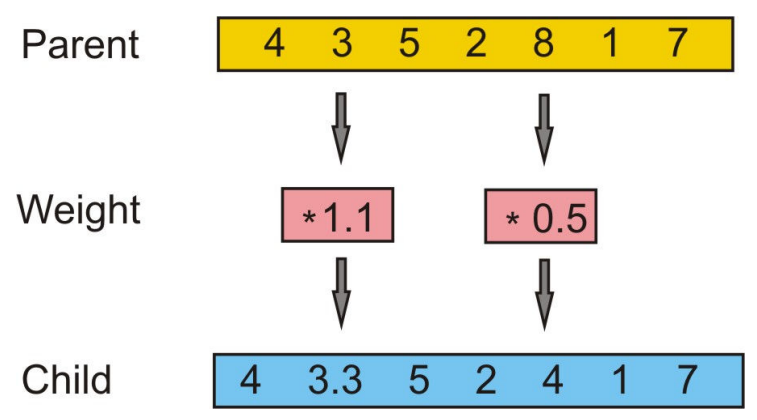

Fig. 6 Scheme of mutation

The cycle of selection, genetic modification and evaluation is repeated until the termination condition is met. The termination condition is defined by:

- finding the searched solution,

- reaching the maximum number of allowed generations (iterations),

- reaching the maximum of allowed computational time,

- reaching the number of generations during which the solution doesn't improve beyond the specified threshold of improvement. This is called a stall of evolutionary algorithm.

\section{Applying evolutionary algorithms to mechanical engineering}

This article shows that evolutionary algorithms can be used in mechanical engineering. They minimize the difference between points of curve obtained experimentally from tensile test and points of curve calculated by Bodner-Partom model $[3,4]$.

A Bodner-Parton model describes the behavior of an elasto-viscoplastic material for small deformations and the whole path of loading and unloading. It is a numerical model including a set of constitutive equations that do not use the yield criterion to separate the elastic area from the inelastic one. It also does not require loading and unloading conditions. This model is based on response properties such as strain rate sensitivity and temperature dependence of inelastic deformation, isotropic and directional hardening for both monotonic and reversed loading, stress saturation under imposed straining, primary and secondary creep, thermal recovery hardening and stress relaxation.

The individuals to be used for the evolutionary algorithm consist of the set parameters for the Bodner-Partom model:

- assumed maximum plastic strain rate $D_{0}$,

- parameter that controls rate sensitivity $n$,

- initial value of isotropic hardening variable $Z_{0}$,

- maximum value of isotropic hardening variable $Z_{1}$,

- rates of isotropic hardening $m_{1}$,

- maximum value of directional hardening variable $Z_{3}$,

- rates of directional hardening $m_{2}$.

Using Matlab, a program based on the Bodner-Partom model and evolutionary algorithms is created. The evolutionary algorithm searches for the best individual with minimal error-difference between the experimental and numerical data. Experimental data was obtained from tensile tests using a test specimen made of an aluminum alloy. The dimensions of this specimen are given in Fig. 7. The sample was pulled by rate $4 \mathrm{~mm} / \mathrm{min}$. 


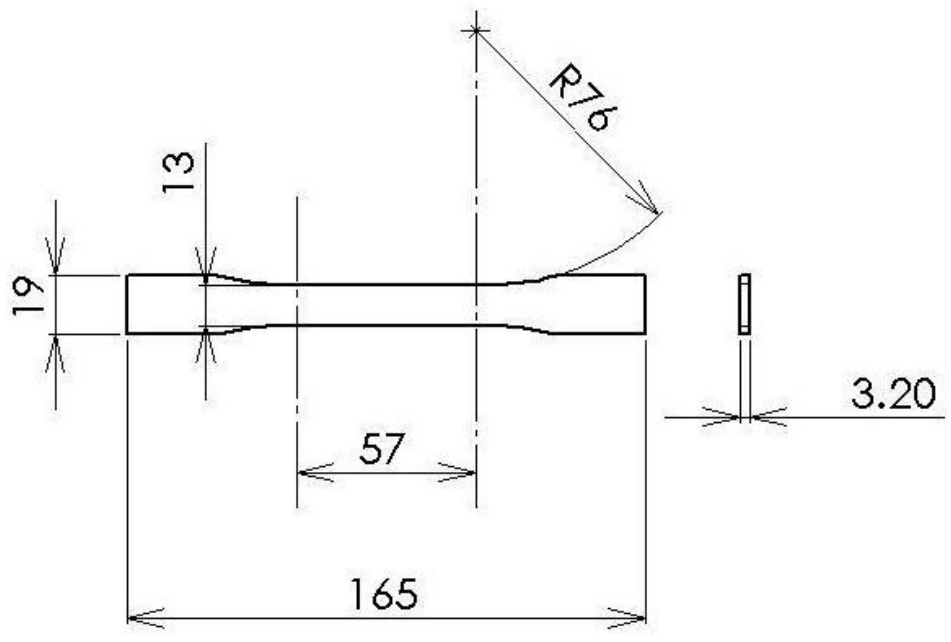

Fig. 7 Dimensions of the specimen

Fig. 8 shows the results where the difference between the experimental and numerical data is compared. The blue dashed line represents the data obtained experimentally and red solid line represents the data determined numerically. The evolutionary algorithm had 24 individuals per generation; the number of elite individuals was 8 . The parents were selected by residual stochastic method and crossover was performed using the scattered method. The program ran for $191.8 \mathrm{~s}$ and was finished at the 81 th generation. The error of the best individual was 0.6156 . It can be seen, that the model does not follow the experimental data precisely.

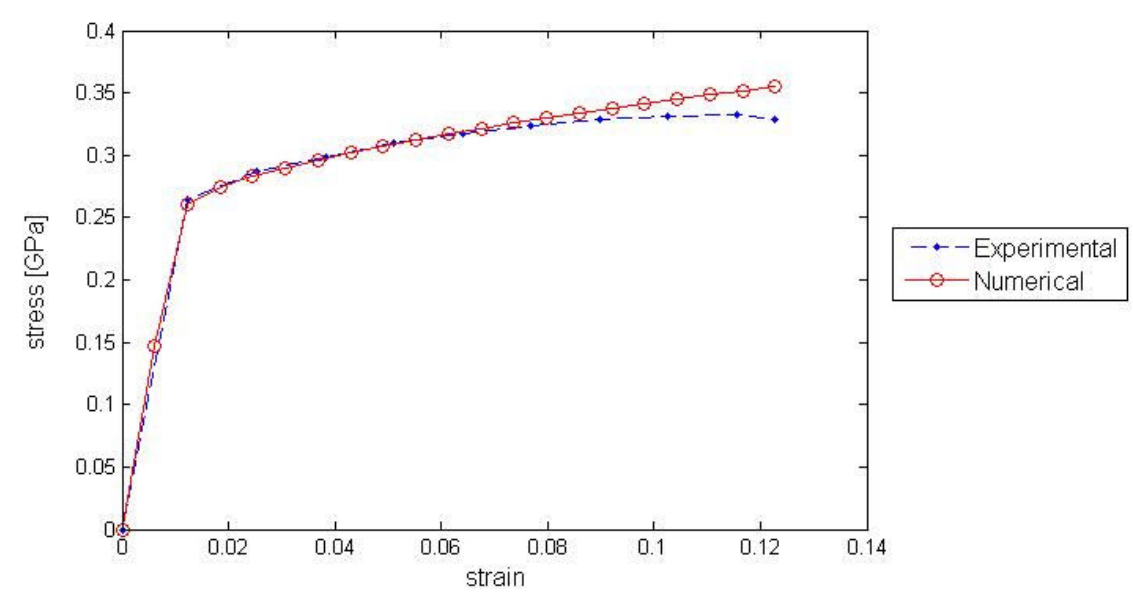

Fig. 8 Diagram illustrating difference between numerical and experimental results

To get a better description of the experimental data by the model, a higher number of generations (300 generations) was chosen in a second run and some termination conditions were disabled to reach that maximum number of generations without stopping prematurely. The program ran for $213.6 \mathrm{~s}$. Using these parameters the error was 0.0377 . The comparison of the experimental data obtained by the tensile test and the numerical data from Bodner-Partom model using modified parameters is shown in Fig. 9. 


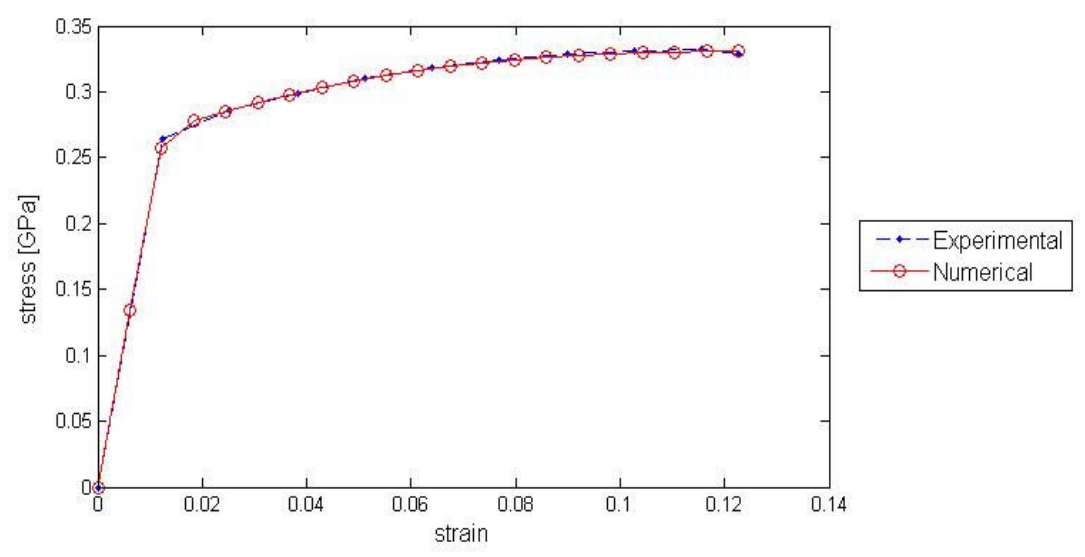

Fig. 9 Diagram illustrating the best approximation of experimental data

\section{Summary}

Evolutionary algorithms are used to solve optimization problems by searching a global minimum. Very good results are obtained by searching for an optimal solution of complex equations that often appear in mechanical engineering. Results of this work show that the settings of the algorithm are very important to get the most appropriate solution. It could also been shown that the genetic algorithms can be very useful in mechanical engineering.

\section{Acknowledgement}

Research presented in this paper was supported by project VEGA 1/1205/12 "Numerical modelling of mechatronic systems."

\section{References}

[1] T. Bäck, Evolutionary Algorithms in Theory and Practice, New York, Oxford University Press, 1996.

[2] J. Bocko, Using Genetic Algorithm to Optimize Whip Constructions (in Slovak), in: Inženírská mechanika, 6, 1997, s. 349-358.

[3] J. Bocko, V. Nohajová, Using Evolutionary Algorithms and Bodner-Partom Model to Determine the Properties of Material, Transcom, Žilina, 2011, 9-th European Conference of Young Research and Scientific Workers, section 6, p. 131-134.

[4] S. R. Bodner, Unified Plasticity for Engineering Applications, New York: Kluwer Academic / Plenum Publishers, 2002.

[5] J. Csontó, M. Palko, Artifficial Life (in Slovak), Košice, Elfa, 2002.

[6] E. Falkenauer, Genetic Algorithms and Grouping Problems, Chichester, John Wiley and Sons, 1998.

[7] D.E. Goldberg, Genetics Algorithms in Search, Optimalization and Machine Learning, in: Addison-Weslwy, 1989.

[8] M. Mach, Evolutionary algorithms, Elements and Principles (in Slovak), Košice, Elfa, 2009, ISBN 978-80-8086-123-0.

[9] V. Nohajová, Identification of Parameters of Bodner-Partom Viscoelastic Material Model (in Slovak), Dissertacion thesis, Košice, 2011. 